\title{
Poblaciones silvestres de Agave angustifolia (Asparagaceae) de Sonora, México: variación morfológica y contenido de azúcares
}

\section{Wild populations of Agave angustifolia (Asparagaceae) from Sonora, Mexico: morphological variation and

\author{
Tania Fragoso-Gadeal (D), Aldo Gutiérrez' (D), Martha L. Coronado² (D), Teresa Terrazas³ (D), Gabriela Ramos-Clamont (ID, \\ Luz Vázquez-Moreno' (D), Osiris Álvarez-Bajo4 (D), Martín Esquedal, (iD)
}

\section{Resumen:}

Antecedentes y Objetivos: "Bacanora" es una bebida espirituosa que emplea como materia prima principalmente las poblaciones silvestres de Agave angustifolia, las cuales requieren estrategias de conservación debido a su sobre aprovechamiento. El objetivo de este trabajo fue evaluar la variación morfológica y el contenido de azúcares reductores totales y fructanos del tallo de $A$. angustifolia proveniente de poblaciones silvestres conservadas en Sonora, México.

Métodos: Se estudiaron tres poblaciones silvestres conservadas de A. angustifolia, en Sonora, México, en 29 variables relacionadas con las características morfológicas, indicadores de madurez fenológica y el contenido de azúcares reductores y fructanos.

Resultados clave: El contenido de fructanos y azúcares reductores totales del tallo presentaron un promedio de 22.6 y $32.7 \mathrm{~g} / 100 \mathrm{~g}$ (base seca) respectivamente; con una variación significativa entre las poblaciones silvestres $(p<0.05)$. De las de 29 variables evaluadas, el estudio de componentes principales indicó que con cuatro componentes se explica 51.9\% de la varianza de los datos. El análisis canónico discriminante de las 16 variables que mostraron mayor relación con los cuatro componentes principales definió el agrupamiento de las poblaciones. Dos funciones canónicas separaron significativamente $(p<0.001)$ a las tres poblaciones silvestres de agave y representaron $100 \%$ de la varianza total.

Conclusiones: Las variables con mayor contribución e importancia relativa asociadas a cada población fueron el desarrollo de la inflorescencia, el contenido de azúcares reductores totales del tallo, así como el tono y la saturación de color de las hojas.

Palabras clave: azúcares reductores, inflorescencia, fructanos, morfología foliar, zonas áridas.

\section{Abstract:}

Background and Aims: "Bacanora" is a spirit drink that uses mainly wild populations of Agave angustifolia as raw material, which require conservation strategies due to their overuse. The aim of this work was to evaluate the morphological variation and the content of total reducing sugars and fructans in the stem of $A$. angustifolia in conserved wild populations from Sonora, Mexico.

Methods: Three conserved wild populations of $A$. angustifolia were studied by 29 variables related to morphological characters, phenological maturity indicators, and the content of reducing sugars and fructans.

Key results: The content of fructans and total reducing sugars in the stem presented an average of 22.6 and $32.7 \mathrm{~g} / 100 \mathrm{~g}$ (dry basis) respectively, with a significant variation among wild populations $(p<0.05)$. Of the 29 variables evaluated, a principal component analysis indicated that $51.9 \%$ of the data variance is explained with four components. A discriminant canonical analysis with the 16 variables that showed the greatest relationship with the four main components defined the grouping of the populations. Two canonical functions significantly separated $(p<0.001)$ the three wild agave populations and represented $100 \%$ of the total variance.

Conclusions: The variables with the greatest contribution and relative importance associated with each population were the development of the inflorescence, the content of total reducing sugars in the stem, as well as the tone and color saturation of leaves.

Key words: drylands, inflorescence, fructans, leaf morphology, reducing sugars.

${ }^{1}$ Centro de Investigación en Alimentación y Desarrollo A.C., Carretera Gustavo Enrique Astiazarán Rosas 46, La Victoria, 83304 Hermosillo, Sonora, México.

${ }^{2}$ Universidad Estatal de Sonora, Av. Ley Federal del Trabajo s/n, Ley 57, 83100 Hermosillo, Sonora, México.

${ }^{3}$ Universidad Nacional Autónoma de México, Instituto de Biología, Circuito Zona Deportiva s/n, Coyoacán, 04510 Cd. Mx., México.

${ }^{4}$ Consejo Nacional de Ciencia y Tecnología-Universidad de Sonora, Blvd. Luis Encinas y Rosales s/n, 83000 Hermosillo, Sonora, México.

${ }^{5}$ Autor para la correspondencia: esqueda@ciad.mx
Recibido: 28 de abril de 2021

Revisado: 26 de mayo de 2021.

Aceptado por Rosario Redonda-Martínez: 25 de octubre de 2021.

Publicado Primero en línea: 12 de noviembre de 2021. Publicado: Acta Botanica Mexicana 128 (2021).

(c) (4) Este es un artículo de acceso abierto bajo la licencia Creative Commons 4.0 Atribución-No Comercial (CC BY-NC 4.0 Internacional).
Citar como: Fragoso-Gadea, T., A. Gutiérrez, M. L. Coronado, T. Terrazas, G. Ramos-Clamont, L. Vázquez-Moreno, Osiris Álvarez-Bajo y M. Esqueda. Poblaciones silvestres de Agave angustifolia (Asparagaceae) de Sonora, México: variación morfológica y contenido de azúcares. Acta Botanica Mexicana 128: e1889. DOI: https://doi.org/10.21829/abm128.2021.1889 


\section{Introducción}

Agave s.s. es un género endémico de América que se distribuye desde el sur de los Estados Unidos de América hasta el norte de Sudamérica (García-Mendoza, 2002). México es el centro de origen y de diversidad natural de Agave L., cuyas especies se presentan principalmente en ecosistemas áridos y semiáridos (García-Mendoza et al., 2019), donde destaca $A$. angustifolia Haw. con la distribución más amplia del género. Parte de esta especie crece de manera silvestre y extensa en la Sierra Madre Occidental, la cual se ubica en el oeste de México a través de Aguascalientes, Chihuahua, Durango, Jalisco, Nayarit, Sinaloa, Sonora y Zacatecas. Este taxón se ha empleado durante más de 300 años en la elaboración de bacanora, bebida espirituosa cuya Denominación de Origen (DOB) comprende 35 municipios de Sonora (Salazar et al., 2015). La materia prima de este destilado proviene básicamente de poblaciones silvestres. Debido a su sobre-aprovechamiento, aunado a la aridez en la sierra y la biología de la especie, las poblaciones se encuentran disminuidas (Sánchez et al., 2020).

Rivera-Lugo et al. (2018) realizaron estudios morfológicos, genéticos y citométricos de $A$. angustifolia silvestre y cultivado de Oaxaca e incluyeron algunos ejemplares silvestres provenientes de Nácori Chico, Sonora. Sus resultados indicaron que los agaves de Sonora correspondían a una entidad diferente de $A$. angustifolia. La taxonomía integrativa (morfología, genética y ecología) esclarecerá en un futuro cercano la identidad de los materiales de la sierra Sonorense.

Con respecto a su biología, $A$. angustifolia se reproduce asexual y sexualmente; a los 6-10 años de vida alcanza su único evento reproductivo sexual, después del cual muere (Gentry, 1982). Su tasa germinativa y de supervivencia son bajas, debido a que generalmente las semillas caen en un medio adverso y pueden ser consumidas por herbívoros (Arzate-Fernández et al., 2016). Estas desventajas son atenuadas a través de la formación vegetativa de vástagos subterráneos y bulbilos en la inflorescencia; sin embargo, está aún lejos de compensar la extracción excesiva. Por lo anterior, el establecimiento de plantaciones es necesario para disminuir la presión sobre las poblaciones silvestres y asegurar la materia prima para la fabricación de bacanora u otros productos (Cervantes-Mendívil et al., 2007; Sánchez et al., 2020). Aunque existen entre 300 y 500 ha sembradas con esta especie de agave, en alrededor de 13 de los 35 municipios de la DOB no hay disponibilidad del recurso biótico como materia prima (CSRB, 2020). Por lo que el futuro de las poblaciones silvestres y la producción del destilado es incierto.

Las condiciones ambientales y la actividad antropogénica mediante la fragmentación del hábitat repercuten sobre la densidad y la variación morfológica de $A$. angustifolia (Ochoa-Meza et al., 2009; Esqueda et al., 2011). Por ejemplo, en el límite este de su distribución en Sonora, donde existe actividad pecuaria con introducción de pastos forrajeros, se han observado ocho grupos morfológicos de poblaciones silvestres de $A$. angustifolia agrupados principalmente por la geometría de la planta, forma foliar y tamaño de espina apical; asimismo, se encontraron individuos que destacan por un mayor peso del tallo y contenido de azúcares (Esqueda et al., 2011). En estudios realizados en Sonora, Gentry (1972) observó que en poblaciones de A. pacifica Trel. en áreas cercanas a la costa, las hojas son cortas y anchas en la parte central; en cambio, en zonas arboladas de la sierra, suelen ser más angostas, largas y laxas; mientras que, en el desierto, A. yaquiana Trel. presentó hojas más cortas, angostas y con mayor número de dientes. Actualmente, A. pacifica y A. yaquiana son sinónimos de $A$. angustifolia (Gentry, 1982).

Debido a la plasticidad morfológica, Gentry (1982) dividió $A$. angustifolia en tres variedades silvestres y cuatro cultivadas. Taxones válidos como A. tequilana F.A.C. Weber y $A$. rhodacantha Trel. se consideran parte del complejo $A$. angustifolia, mientras que alrededor de 20 especies están en sinonimia con $A$. angustifolia.

El objetivo de esta investigación es evaluar la variación morfológica y el contenido de azúcares reductores totales y fructanos del tallo en poblaciones silvestres conservadas de $A$. angustifolia, ubicadas al centro de su distribución en Sonora, México, con la finalidad de coadyuvar en la definición de criterios para el manejo del agave.

\section{Materiales y Métodos}

\section{Poblaciones estudiadas}

Se seleccionaron tres poblaciones silvestres conservadas de $A$. angustifolia localizadas en ranchos pertenecientes 


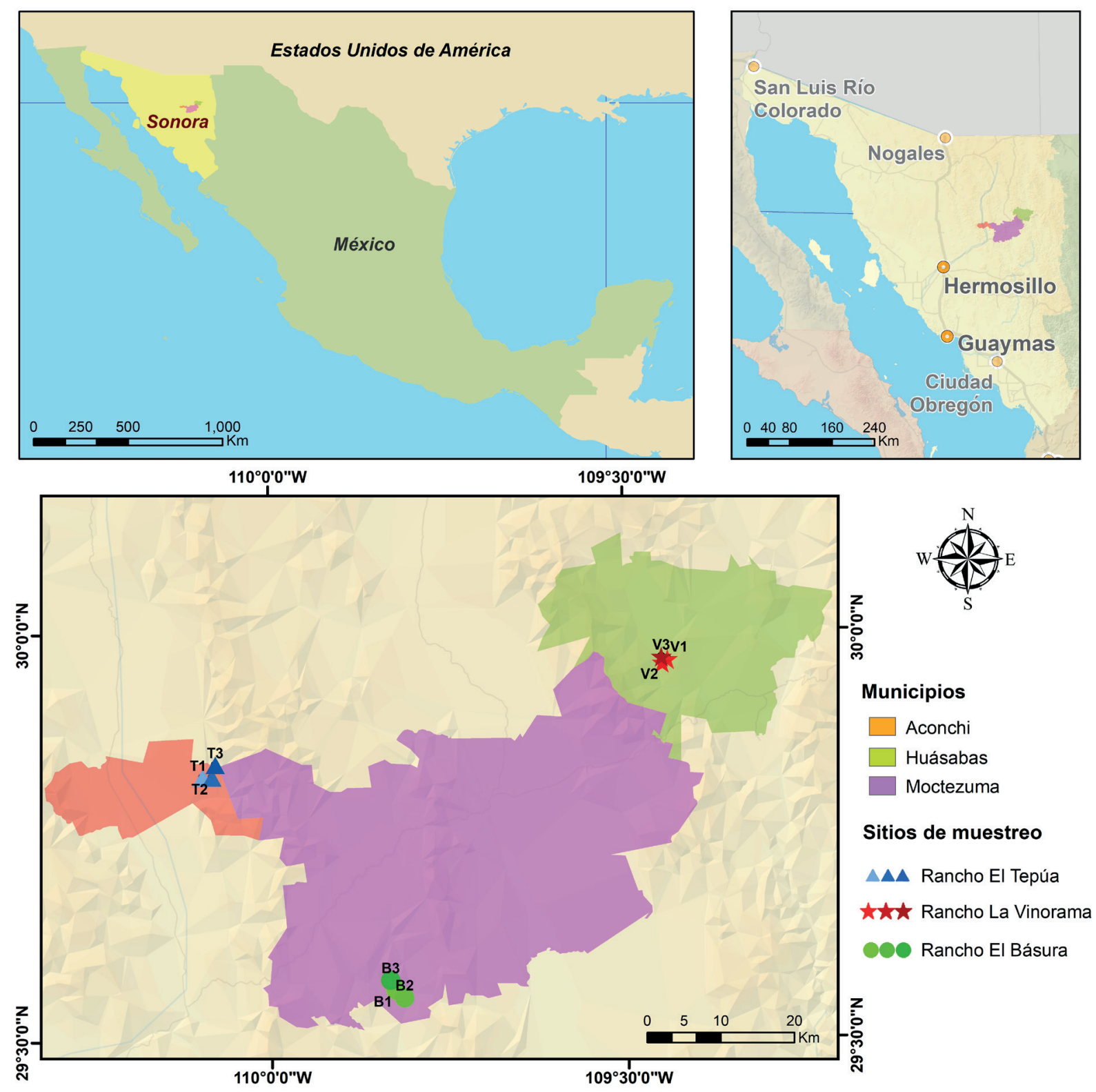

Figura 1: Ubicación de las poblaciones de Agave angustifolia Haw. estudiadas en Sonora, México.

a tres municipios de Sonora, México, las cuales se representan en el mapa de la Figura 1, el cual se elaboró con el programa ArcGIS v. 10.0 (ESRI, 2011). En cada una de las poblaciones se delimitaron tres cuadrantes de $50 \times 50 \mathrm{~m}$ $\left(2500 \mathrm{~m}^{2}\right)$, considerando el tipo de vegetación y los límites de distribución de las poblaciones de $A$. angustifolia. La caracterización poblacional y morfológica se realizó durante las estaciones de primavera y verano.

Población 1: Municipio Aconchi, Rancho El Tepúa $(\mathrm{T})$, cuadrante uno (T1): $29^{\circ} 49^{\prime} 21^{\prime \prime} \mathrm{N}, 110^{\circ} 5^{\prime} 42^{\prime \prime} \mathrm{O}$; cuadrante 2 (T2): $29^{\circ} 49^{\prime} 22^{\prime \prime} \mathrm{N}, 110^{\circ} 4^{\prime} 55^{\prime \prime} \mathrm{O}$; cuadrante 3 (T3): $29^{\circ} 50^{\prime} 25^{\prime \prime} \mathrm{N}, 110^{\circ} 4^{\prime} 24^{\prime \prime} \mathrm{O}$. El rancho presentó un tipo de vegetación de matorral subtropical con clima estepario semiseco semicálido (BS1hw(x')) a una altitud de 987 m s.n.m. y suelos litosol, regosol y vertisol (INEGI, 2001; 2008).

Población 2: Municipio Huásabas, Rancho La Vinorama (V), cuadrante 1 (V1): $29^{\circ} 57^{\prime} 45^{\prime \prime} \mathrm{N}, 109^{\circ} 26^{\prime} 21^{\prime \prime O}$; cuadrante 2 (V2): $29^{\circ} 57^{\prime} 30^{\prime \prime} \mathrm{N}, 109^{\circ} 26^{\prime} 47^{\prime \prime O}$; cuadrante 3 (V3): $29^{\circ} 57^{\prime} 57^{\prime \prime} \mathrm{N}, 109^{\circ} 26^{\prime} 51^{\prime \prime} \mathrm{O}$. El rancho mostró un tipo de vegetación de mezquital con un clima estepario semiseco semicálido (BS1hw(x')) a una altitud de 801 m s.n.m. y suelos litosol y regosol (INEGI, 2001; 2008). 
Población 3: Municipio Moctezuma, Rancho El Básura (B), cuadrante 1 (B1): 29³3'37"N, 10949'32"O; cuadrante 2 (B2): $29^{\circ} 33^{\prime} 3^{\prime \prime} \mathrm{N}, 109^{\circ} 48^{\prime} 49^{\prime \prime} \mathrm{O}$; cuadrante 3 (B3): $29^{\circ} 34^{\prime} 20^{\prime \prime} \mathrm{N}, 109^{\circ} 49^{\prime} 49^{\prime \prime} \mathrm{O}$. El rancho presentó un tipo de vegetación de matorral subtropical con un clima estepario semiseco semicálido $\left(B S 1 h w\left(x^{\prime}\right)\right)$ a una altitud de $756 \mathrm{~m}$ s.n.m. y suelo litosol (INEGI, 2001; 2008).

\section{Caracterización poblacional y morfológica}

En los nueve cuadrantes se contabilizó el número de plantas de $A$. angustifolia para estimar su densidad, clasificándolas de acuerdo con su tipo de crecimiento (independiente y agrupado). La presencia de una planta madre única se consideró como independiente y algunos atributos ecológicos como el número de hijuelos se cuantificó con base en agaves adultos para una información más precisa, de tal forma que, dos o más agaves adultos juntos se clasificaron como agrupados. Los criterios de inclusión para la caracterización morfológica fueron: talla $>1 \mathrm{~m}$, buen estado general, es decir, sin ningún indicio o síntoma aparente de enfermedad y presencia de los siguientes indicadores de madurez: grado de desarrollo y estado de la inflorescencia, hojas laterales amarillas y/o acortamiento de hojas centrales (Fig. 2). Debido a que el crecimiento y desarrollo de los agaves es multifactorial, resulta imposible definir la edad de especímenes silvestres, siendo arbitrario el valor de la talla $>1 \mathrm{~m}$. Con base en estudios previos (Colunga-GarcíaMarín et al., 1996; Colunga-GarcíaMarín y May-Pat, 1997; Rodríguez-Garay et al., 2009) se analizaron las características poblacionales: magueyes adultos independientes o agrupados, diámetro y altura de la roseta, número de hijuelos, nodricismo, considerando la distancia y orientación cardinal con respecto a la planta asociada e indicadores de madurez.

De cada planta se tomaron tres hojas centrales completas para establecer las siguientes relaciones: peso seco/ peso fresco, longitud total/ancho de la base foliar, longitud total/ancho de la porción media foliar y número de dientes/longitud de la hoja. Se cuantificó la distancia entre dientes, longitud y ancho basal de los dientes, distancia de la espina apical al primer diente, así como longitud y ancho basal de la espina apical. Para la determinación de color se emplearon los parámetros de luminosidad ( $\left.L^{*}\right)$, ángulo de matiz o tono ( ${ }^{\circ} \mathrm{Hue}$ ) y pureza (Croma) del sistema CIE
L*a*b* (CIE, 2018), con un colorímetro portátil Minolta CR300 (Konica Minolta Inc., New Jersey, EUA). La determinación de color se realizó en la región basal, media y apical del haz de cada hoja, registrando los valores de $a^{*}, b^{*}$ y $L^{*}$; las fórmulas para la estimación del tono y la pureza fueron: 'Hue $=(\operatorname{arcTan}(b * / a *))$ y Croma $=(a * 2+b * 2)-1 / 2$.

\section{Cuantificación de azúcares}

Como complemento a los indicadores de madurez, se estimó el contenido promedio de sólidos solubles totales de las hojas mediante la determinación de ${ }^{\circ}$ Brix. Se extrajo una muestra de la porción basal de cada hoja con un horadador MW-26A (Che Scientific Co., Kwai Chung, Hong Kong) y se exprimió el jugo en un refractómetro portátil ATAGO PR101 (ATAGO Inc., Bellevue, EUA). Para la cuantificación de azúcares se tomaron las medidas y el peso en fresco del tallo de las plantas consideradas en el análisis morfológico y que presentaron los mejores indicadores de madurez, teniendo en cuenta la inflorescencia ausente, en desarrollo u eliminada por los productores uno o dos años antes de realizar el estudio. Además, se seleccionó una porción longitudinal representativa del tallo (15-25\% según el tamaño) y se determinaron ${ }^{\circ}$ Brix de la región basal, media y apical del tallo.

Después, las muestras se redujeron a harina para estimar el porcentaje de azúcares reductores (AR) y el contenido de fructanos. Para ello, se cortaron finamente y se secaron en una estufa Micro-Pack Mod. MP500 (Enviro-Pak, Clakamas, EUA) a $50{ }^{\circ} \mathrm{C}$ por $72 \mathrm{~h}$. Se estimó el peso seco total para obtener la proporción peso seco/peso fresco; luego los trozos se pulverizaron en un molino eléctrico Wiley Mod. 4 (Thomas Scientific, Swedesboro, EUA) con mallas de 9.5 a $0.2 \mathrm{~mm}$ de diámetro. Para las determinaciones se realizaron extractos acuosos de soluciones conteniendo 2 $\mathrm{mg} / \mathrm{ml}$ de harina, calentadas a $80^{\circ} \mathrm{C}$, durante $15 \mathrm{~min}$ (AOAC 999.03-2005).

El contenido de AR se cuantificó por triplicado con el método del ácido dinitrosalicílico (DNS), con un espectrofotómetro Cary 1 (Varian Inc., Victoria, Austria) a una longitud de onda de $540 \mathrm{~nm}$ (Chaplin, 1986). Los fructanos se analizaron por duplicado con un método enzimático/ colorimétrico (AOAC 999.03-2005 y AACC 32-32.01), con el procedimiento para fructano de Megazyme $^{\circledR}$; las medicio- 

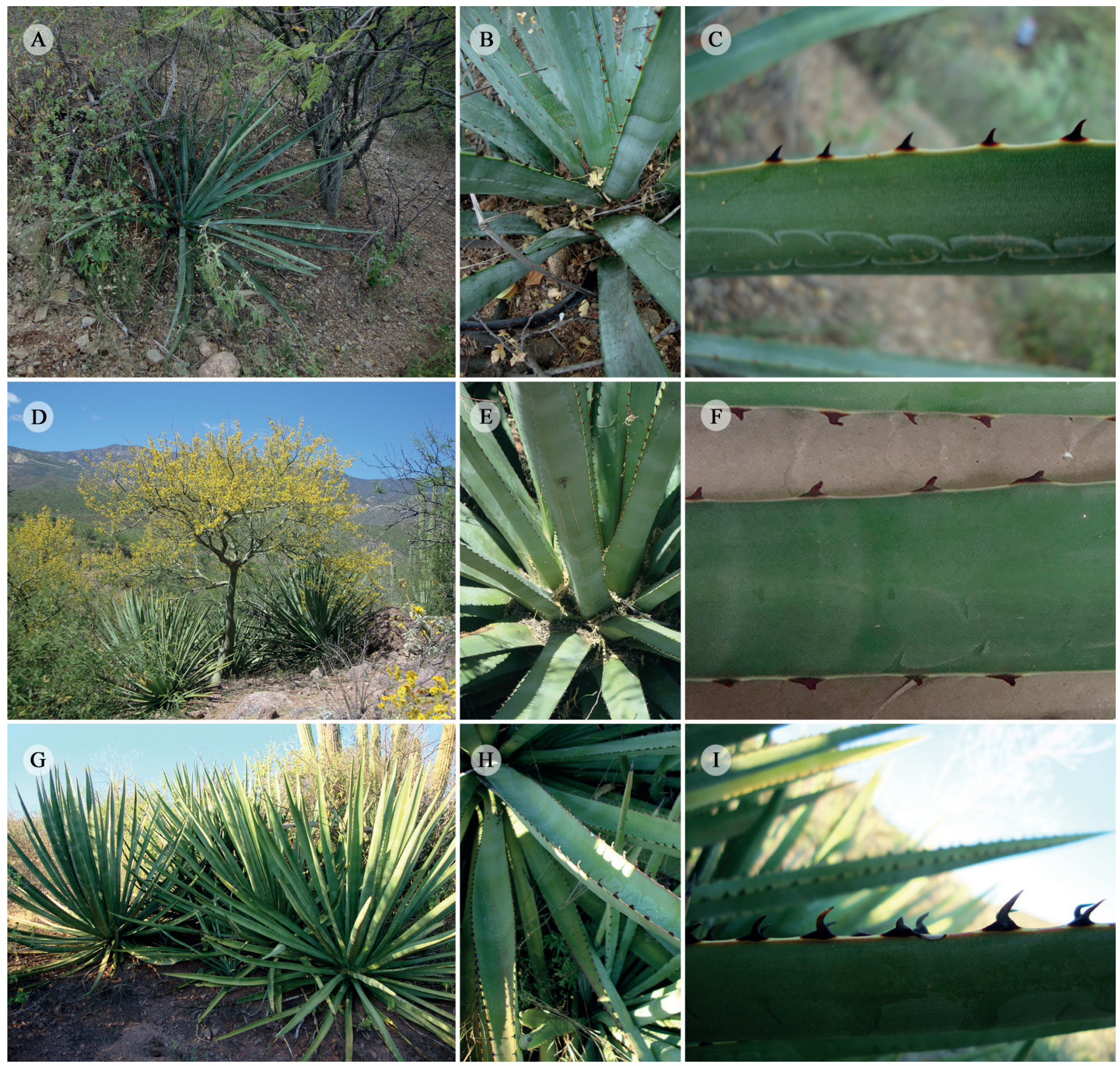

Figura 2: Características morfológicas generales de Agave angustifolia Haw. en los diferentes sitios de muestreo en Sonora, México. A-C. Rancho El Tepúa; D-F. Rancho La Vinorama; G-I. Rancho El Básura.

nes se efectuaron en el espectrofotómetro a una longitud de onda $410 \mathrm{~nm}$. Para la estimación de azúcares reductores totales (ART) se utilizó como factor de conversión el paso de anhidrofructosa a fructosa libre, mediante la siguiente fórmula: contenido de fructanos/0.906, más la cantidad de AR (AOAC 999.03-2005) (Megazyme, 2011).

\section{Análisis estadístico}

Se realizó estadística descriptiva para todos los parámetros y análisis de varianza en las variables continuas poblacionales, morfológicas y contenido de azúcares con el paquete de software NCSS (Hintze, 2001). Se consideró un nivel de confianza de $95 \%$ y para la comparación entre medias se utilizó el método de Tukey-Kramer. Con la finalidad de identificar aque- 
Ilas variables que permiten explicar el mayor porcentaje de la varianza se hizo un análisis de componentes principales (ACP). Para determinar los patrones de agrupamiento entre las poblaciones, se empleó un análisis canónico discriminante con aquellos caracteres indicados por el ACP, con el método de distancia al cuadrado pareada entre grupos (PSDBG, por sus siglas en inglés, Pairwise Squared Distance Between Groups) y la diferenciación entre poblaciones se evaluó mediante la distancia de Mahalanobis (D2), ambos con el programa estadístico SAS v. 9.2 (SAS, 2008).

\section{Resultados}

\section{Análisis morfológico}

El total de agaves que cumplieron el criterio de inclusión para realizar el análisis morfológico, fue 567 (Básura), 542 (Vinorama) y 369 (Tepúa), de los cuales se seleccionaron aleatoriamente ca. 3\%, siendo 44 el total de los individuos evaluados (Básura 20, Tepúa 12 y Vinorama 12). Estos organismos adultos tuvieron una altura y un diámetro promedio global de 1.4 y 2.1 m respectivamente.

La longitud de las hojas varió de 72 a $164 \mathrm{~cm}$, con un promedio de $113 \mathrm{~cm}$, mientras que el ancho de 2.5 a $10 \mathrm{~cm}$, con un promedio de $5.7 \mathrm{~cm}$. En el Cuadro 1 se presentan los valores promedio de las variables morfológicas foliares por población, los cuales no fluctuaron significativamente $(p<0.05)$, excepto en algunas como la longitud de los dientes, ancho basal de la espina apical y color foliar (luminosidad, tono y saturación).

\section{Análisis poblacional}

En el análisis poblacional se incluyeron todos los individuos por cuadrante. La densidad más alta se registró en el cuadrante Básura 1 con 1268 individuos ha-1, seguida de

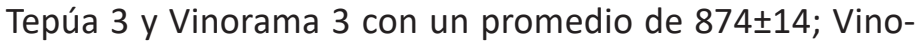
rama 1, 2 y Básura 3 con 651 24 , y Tepúa 1, 2 y Básura 2 con $315 \pm 33$ individuos ha-1. La distribución en los sitios no es continua, sino agrupada en $91 \%$ de los agaves y $9 \%$ restante con plantas madre independientes. Cuando se realizó el trabajo en campo, la única actividad observada en las poblaciones fue la eliminación de la inflorescencia en los ranchos El Básura (0\%), La Vinorama (42\%) y El Tepúa (100\%), sin extracción aún de especímenes.
De los individuos, $82 \%$ se observó bajo la protección del dosel de diferentes árboles caducifolios. La especie asociada con mayor frecuencia fue Bursera laxiflora S. Watson en Básura, Vachellia campechiana (Mill.) Seigler \& Ebinger en Tepúa y especies de Prosopis L. en Vinorama. La distancia promedio entre el agave y la planta nodriza fue de $0.8 \mathrm{~m}$, mientras que su orientación cardinal varió, siendo la posición más frecuente el sur en Básura, noreste en Tepúa y suroeste en Vinorama (Cuadro 2).

\section{Contenido de azúcares}

Las características generales del tallo y el contenido de azúcares se resumen en el Cuadro 3. El diámetro promedio del tallo fue de $26.0 \pm 4.7 \mathrm{~cm}$, mientras que el peso fresco y seco de $12.4 \pm 6.7$ y $3.6 \pm 2.3 \mathrm{~kg}$, respectivamente; la biomasa representó alrededor de $30 \%$. El porcentaje de ${ }^{\circ}$ Brix varió entre poblaciones ( $p<0.05)$. Los sólidos solubles totales de la base foliar de Vinorama tuvieron el valor más alto con 10.9, siendo la media general de $8.7 \pm 3.1$. El porcentaje de sólidos solubles totales del tallo registró valores de $30.9,21.9$ y $12.3 \%{ }^{\circ}$ Brix, para Vinorama, Básura y Tepúa, respectivamente $(p<0.05)$. El contenido de fructanos fluctuó de 5.1 a $61.2 \mathrm{~g} / 100 \mathrm{~g}$ base seca (BS), con un promedio poblacional de 29.7, 20.3 y 13.1\% para Básura, Vinorama y Tepúa, respectivamente $(p<0.05)$. El contenido promedio de fructanos fue de $35.2 \mathrm{~g} / 100 \mathrm{~g}$ BS en los especímenes de las tres poblaciones silvestres con la inflorescencia eliminada, mientras que el valor se redujo alrededor de un $50 \%$ en los agaves $\sin (16.9 \mathrm{~g} / 100 \mathrm{~g} \mathrm{BS})$ y con ( $18.0 \mathrm{~g} / 100 \mathrm{~g} \mathrm{BS})$ inflorescencia en desarrollo. El porcentaje de ART del tallo cambió también entre las poblaciones $(p<0.05)$, siguiendo la misma tendencia que en fructanos. La correlación entre ${ }^{\circ}$ Brix y el contenido de ART y fructanos fue de 0.62 y 0.65 , respectivamente.

\section{Análisis de componentes principales (ACP)}

El ACP con 29 variables mostró que cuatro componentes explican $51.9 \%$ de la variabilidad de los datos. Con base en los valores de los vectores de cada una de ellas se identificaron las variables más importantes que definen el primer componente: a) ${ }^{\circ}$ Brix del tallo, b) relación peso seco/peso fresco del tallo, c) ART, d) tono color foliar, e) grado de desa- 
Cuadro 1: Características morfológicas de Agave angustifolia Haw., en tres municipios del estado de Sonora, México. * Literal distinta entre columnas indica diferencia significativa Tukey-Kramer $\mathrm{p}<0.05$. ${ }^{* *}$ Media \pm desviación estándar y adjetivo de color más frecuente, ${ }^{* * * A-V: ~ a m a r i l l o-v e r d o s o ; ~}$ V-Ag: verde-amarillo grisáceo; A-Vgc: amarillo-verde grisáceo claro; V-Ao: verde-amarillo opaco.

\begin{tabular}{|c|c|c|c|c|}
\hline Variables & El Básura* & El Tepúa & La Vinorama & Promedio Total** \\
\hline Relación peso seco/peso fresco foliar (g) & $0.2^{\mathrm{a}}$ & $0.2 \mathrm{a}$ & $0.2 \mathrm{a}$ & $0.2 \pm 0.04$ \\
\hline Longitud/ancho base foliar (cm) & $18.4^{\mathrm{a}}$ & $21.1 \mathrm{a}$ & $18.5 a$ & $19.2 \pm 4.2$ \\
\hline Longitud/ancho porción media foliar (cm) & $21.5^{\mathrm{a}}$ & $21.3 a$ & $20.1 a$ & $21.0 \pm 5.0$ \\
\hline Número dientes/longitud foliar (cm) & $0.9^{\mathrm{a}}$ & 1.0a & $0.9 a$ & $0.9 \pm 0.2$ \\
\hline Distancia entre dientes centrales (cm) & $2.1^{\mathrm{a}}$ & $2.2 \mathrm{a}$ & $1.9 a$ & $2.1 \pm 0.9$ \\
\hline Longitud dientes (cm) & $0.5^{\mathrm{a}}$ & $0.6 b$ & $0.5 a$ & $0.5 \pm 0.2$ \\
\hline Ancho dientes $(\mathrm{cm})$ & $0.9^{\mathrm{a}}$ & $0.8 a$ & $0.8 a$ & $0.8 \pm 0.3$ \\
\hline Distancia del primer diente a la espina apical $(\mathrm{cm})$ & $5.0^{\mathrm{a}}$ & $6.4 a$ & $6.5 a$ & $5.8 \pm 2.5$ \\
\hline Longitud de espina apical (cm) & $2.2^{\mathrm{a}}$ & $2.3 a$ & $2.7 a$ & $2.3 \pm 0.7$ \\
\hline Ancho basal de espina apical $(\mathrm{cm})^{*}$ & $0.5^{\mathrm{a}}$ & $0.55 a b$ & $0.6 b$ & $0.6 \pm 0.1$ \\
\hline Luminosidad del color (L)* & $47.9^{\mathrm{a}}$ & $44.8 \mathrm{a}$ & $52.9 \mathrm{~b}$ & $48.4 \pm 5.0$ \\
\hline Tono del color $\left({ }^{\circ} \mathrm{Hue}\right)^{*}$ & $125.1^{\mathrm{a}}$ & $129.2 b$ & $123.5 a$ & $125.8 \pm 4.1$ \\
\hline Saturación de color (Croma)* & $33.0^{\mathrm{b}}$ & $26.5 a$ & $23.5 a$ & $28.6 \pm 6.6$ \\
\hline Adjetivo de color*** & $A-V$ & $\mathrm{~V}-\mathrm{Ag}$ & $A-\operatorname{Vgc}$ & V-Ao \\
\hline
\end{tabular}

Cuadro 2: Características poblacionales de Agave angustifolia Haw., en tres municipios del estado de Sonora, México. *Literal distinta entre columnas indica diferencia significativa Tukey-Kramer $p<0.05$. **Media \pm desviación estándar u orientación más frecuente.

\begin{tabular}{|c|c|c|c|c|c|}
\hline \multicolumn{2}{|l|}{ Variables } & El Básura* & El Tepúa & La Vinorama & Promedio Total** \\
\hline \multicolumn{2}{|c|}{ Diámetro de planta (m) } & $2.1^{\mathrm{a}}$ & $1.9^{\mathrm{a}}$ & $2.3^{\mathrm{a}}$ & $2.1 \pm 0.5$ \\
\hline \multicolumn{2}{|l|}{ Altura de planta (m) } & $1.4^{\mathrm{a}}$ & $1.4^{\mathrm{a}}$ & $1.5^{\mathrm{a}}$ & $1.4 \pm 0.3$ \\
\hline \multicolumn{2}{|c|}{ Relación diámetro/altura } & $1.6^{\mathrm{a}}$ & $1.4^{\mathrm{a}}$ & $1.5^{\mathrm{a}}$ & $1.5 \pm 0.3$ \\
\hline \multicolumn{2}{|l|}{ Número de hijuelos } & $1.8^{\mathrm{a}}$ & $1.9^{\mathrm{a}}$ & $2.6^{\mathrm{a}}$ & $2.0 \pm 1.8$ \\
\hline \multicolumn{2}{|c|}{ Orientación cardinal más frecuente } & $\mathrm{s}$ & $\mathrm{NE}$ & so & $\mathrm{S}$ \\
\hline \multirow{2}{*}{ Nodricismo } & Con nodriza (\%) & 75 & 100 & 75 & 82 \\
\hline & Expuesto (\%) & 25 & 0 & 25 & 18 \\
\hline \multicolumn{2}{|c|}{ Posición frecuente respecto a nodriza principal } & $E, N$ & E, SE & 0 & $N, E, O$ \\
\hline \multicolumn{2}{|c|}{ Distancia de nodriza principal (m) } & $0.8^{\mathrm{a}}$ & $0.8^{\mathrm{a}}$ & $0.7^{\mathrm{a}}$ & $0.8 \pm 0.7$ \\
\hline \multirow{2}{*}{ Tipo de crecimiento } & Individual (\%) & 5 & 17 & 8 & 9 \\
\hline & Agrupado (\%) & 95 & 83 & 92 & 91 \\
\hline \multicolumn{2}{|c|}{ Orientación cardinal más frecuente } & $\mathrm{s}$ & NE & so & $\mathrm{s}$ \\
\hline \multirow{3}{*}{$\begin{array}{l}\text { Desarrollo de la } \\
\text { inflorescencia }\end{array}$} & Sin quiote (\%) & $0^{\mathrm{b}}$ & $0^{\mathrm{b}}$ & $58^{\mathrm{a}}$ & 16 \\
\hline & Temporada anterior (\%) & $30^{\mathrm{b}}$ & $0^{\mathrm{b}}$ & $42^{\mathrm{a}}$ & 25 \\
\hline & Nuevo (\%) & $70^{\mathrm{b}}$ & $100^{\mathrm{b}}$ & $0^{\mathrm{a}}$ & 59 \\
\hline \multirow{2}{*}{$\begin{array}{l}\text { Estado de la } \\
\text { inflorescencia }\end{array}$} & Completo (\%) & 70 & 100 & 0 & 70 \\
\hline & Cortado (\%) & 30 & 0 & 100 & 30 \\
\hline
\end{tabular}


Cuadro 3: Características físicas y azúcares del tallo de Agave angustifolia Haw., en tres municipios del estado de Sonora, México. * Literal distinta entre columnas indica diferencia significativa Tukey-Kramer $p<0.05$. ${ }^{*}$ Media \pm desviación estándar.

\begin{tabular}{|c|c|c|c|c|}
\hline Variables & El Básura* & El Tepúa & La Vinorama & $\begin{array}{r}\text { Promedio } \\
\text { Total** }\end{array}$ \\
\hline Sólidos solubles totales base foliar ( $\left.\%{ }^{\circ} \mathrm{Brix}\right)$ & $8.5^{\mathrm{ab}}$ & $6.7^{\mathrm{a}}$ & $10.9^{\mathrm{b}}$ & $8.7 \pm 3.1$ \\
\hline Diámetro tallo $(\mathrm{cm})$ & $26.3^{\mathrm{a}}$ & $25.0^{\mathrm{a}}$ & $26.5^{\mathrm{a}}$ & $26.0 \pm 4.7$ \\
\hline Peso fresco tallo (kg) & $12.2^{\mathrm{a}}$ & $11.3^{\mathrm{a}}$ & $13.7^{\mathrm{a}}$ & $12.4 \pm 6.7$ \\
\hline Peso seco tallo $(\mathrm{kg})$ & $3.6^{\mathrm{a}}$ & $2.7^{\mathrm{a}}$ & $4.7^{\mathrm{a}}$ & $3.6 \pm 2.3$ \\
\hline Relación peso seco/peso fresco tallo & $0.3^{\mathrm{ab}}$ & $0.2^{\mathrm{a}}$ & $0.4^{b}$ & $0.3 \pm 0.1$ \\
\hline Sólidos solubles totales tallo ( $\%{ }^{\circ} \mathrm{Brix}$ ) & $21.9^{b}$ & $12.3^{\mathrm{a}}$ & $30.9^{c}$ & $21.7 \pm 10.2$ \\
\hline Fructanos totales (g/100 g BS) & $29.7^{b}$ & $13.1^{\mathrm{a}}$ & $20.3^{\mathrm{ab}}$ & $22.6 \pm 13.8$ \\
\hline Azúcares reductores (AR) (g/100 g BS) & $6.8^{\mathrm{a}}$ & $8.7^{\mathrm{a}}$ & $7.6^{\mathrm{a}}$ & $7.5 \pm 2.7$ \\
\hline Azúcares reductores totales (ART) (g/100 g BS) & $39.6^{b}$ & $23.2^{\mathrm{a}}$ & $30.0^{\mathrm{ab}}$ & $32.5 \pm 13.9$ \\
\hline
\end{tabular}

rrollo de la inflorescencia, f) ${ }^{\circ}$ Brix de la base foliar y g) estado de la inflorescencia. El segundo componente está representado por las variables: h) altura, i) diámetro de planta, j) peso fresco del tallo y k) relación peso seco/peso fresco foliar. En el tercero se tiene: I) croma color foliar, $\mathrm{m}$ ) ancho basal y $n$ ) longitud de espina apical. El cuarto componente corresponde a: o) tipo de crecimiento y p) longitud total/ ancho de sección media foliar (Fig. 2).

\section{Análisis canónico discriminante (ACD)}

Las 16 variables seleccionadas en el ACP se sometieron a un ACD para determinar si existían patrones de agrupamiento entre las poblaciones. El análisis mostró que dos funciones canónicas permiten una separación definida de las tres poblaciones silvestres (Cuadro 4, Fig. 3) con una contribución significativa (Lambda de Wilks $\mathrm{p}<0.001$ ). Al contrastar la diferencia entre cada par de poblaciones mediante la distancia de Mahalanobis (D2), todas las combinaciones resultaron significativas $(p<0.05)$. Las poblaciones con mayor cercanía fueron Tepúa y Básura, con una distancia entre sus centroides de 7.9, mientras que estas tuvieron 96.4 y 97.8 respectivamente con la población Vinorama (Fig. 3), lo cual indica que estos especímenes se distinguen en las características evaluadas de Tepúa y Básura.

En el Cuadro 4 se presentan los coeficientes estandarizados de las dos funciones canónicas con la contribución conjunta de las variables evaluadas y la importancia relati- va de cada una. La primera función canónica separa las poblaciones Tepúa y Básura del área Vinorama (Fig. 3), debido al grado de desarrollo de la inflorescencia, así como el tono y la saturación de color foliar. En el total de los individuos muestreados de la población Tepúa, la inflorescencia se eliminó al emerger. En Vinorama, 58\% de los agaves evaluados no presentó y en $42 \%$ restante, se cortó uno o dos años antes de realizar el estudio. En el área Básura, 70\% de los especímenes cosechados tenía inflorescencia reciente y $30 \%$ con el quiote desarrollado. Con relación al color foliar, los especímenes de Básura tienden hacia amarillo-verdoso ligeramente más vivo que en las otras poblaciones, donde predomina el verde sobre el amarillo, con un valor de croma más apagado. La segunda función canónica distingue entre las poblaciones Tepúa y Básura (Fig. 3), debido principalmente al contenido de ART con valores de 39.6, 30.0 y $23.2 \%$ para Básura, Vinorama y Tepúa, respectivamente (Cuadro 3).

\section{Discusión}

\section{Análisis poblacional}

Esta evaluación estableció las bases para una selección representativa y aleatoria de individuos con madurez fenológica en las tres poblaciones silvestres. En el estudio de Ochoa-Meza et al. (2009) en poblaciones manejadas de $A$. angustifolia en el límite este de la distribución de la especie en Nácori Chico, Sonora, la densidad fluctuó de 199 a 503 
Cuadro 4: Coeficientes canónicos estandarizados de las principales variables evaluadas en Agave angustifolia Haw., en poblaciones silvestres de tres municipios del estado de Sonora, México.

\begin{tabular}{|c|c|c|}
\hline Variable & $\begin{array}{l}\text { Función } \\
\text { canónica } 1\end{array}$ & $\begin{array}{l}\text { Función } \\
\text { canónica } 2\end{array}$ \\
\hline Tono de color foliar & 1.55 & -0.07 \\
\hline Saturación de color foliar & 1.50 & 0.50 \\
\hline $\begin{array}{l}\text { Relación peso seco/peso fresco } \\
\text { foliar }\end{array}$ & 0.88 & 0.36 \\
\hline $\begin{array}{l}\text { Longitud total/ancho de porción } \\
\text { media foliar }\end{array}$ & -0.22 & -0.05 \\
\hline Ancho basal de espina apical & 0.07 & -0.25 \\
\hline Longitud de espina apical & -0.66 & 0.01 \\
\hline $\begin{array}{l}\text { Sólidos solubles totales ('Brix) basal } \\
\text { foliar }\end{array}$ & -1.01 & 0.40 \\
\hline Diámetro de planta & 0.56 & 0.65 \\
\hline Altura de planta & -0.25 & 0.34 \\
\hline Peso fresco de tallo & -0.66 & -0.87 \\
\hline $\begin{array}{l}\text { Relación peso seco/peso fresco de } \\
\text { tallo }\end{array}$ & 0.82 & -0.66 \\
\hline $\begin{array}{l}\text { Sólidos solubles totales }\left({ }^{\circ} \text { Brix }\right) \text { de } \\
\text { tallo }\end{array}$ & -1.02 & 0.43 \\
\hline Azúcares reductores totales & 1.42 & 1.26 \\
\hline $\begin{array}{l}\text { Grado de desarrollo de la } \\
\text { inflorescencia }\end{array}$ & 2.25 & -0.60 \\
\hline Estado de la inflorescencia & -0.05 & -0.59 \\
\hline Tipo de crecimiento & -0.09 & 0.46 \\
\hline
\end{tabular}

individuos ha-1 y en el presente trabajo de 315 a 1268. Las diferencias podrían deberse a las condiciones ambientales, la dinámica del agave y el grado de fragmentación por las actividades antropogénicas. En la localidad bajo manejo y con la mayor densidad se observó un microclima propiciado por un lomerío suave, una mayor cobertura vegetal y un menor grado de perturbación, lo cual podría favorecer la distribución del agave, más aún que estaba principalmente asociado con árboles y arbustos como Vachellia campechiana, Fouquieria macdougalii Nash, Prosopis spp. y Sebastiania bilocularis S. Watson.

En un estudio poblacional de $A$. angustifolia en un bosque tropical seco se encontró de forma similar una densidad mayor en el sitio conservado vs. alterado de 5.9 y 2.4 agaves $/ 100 \mathrm{~m}^{2}$ respectivamente $(\mathrm{p}<0.001)$ (Arias-Medellín et al., 2016). Las poblaciones silvestres de $A$. angustifolia

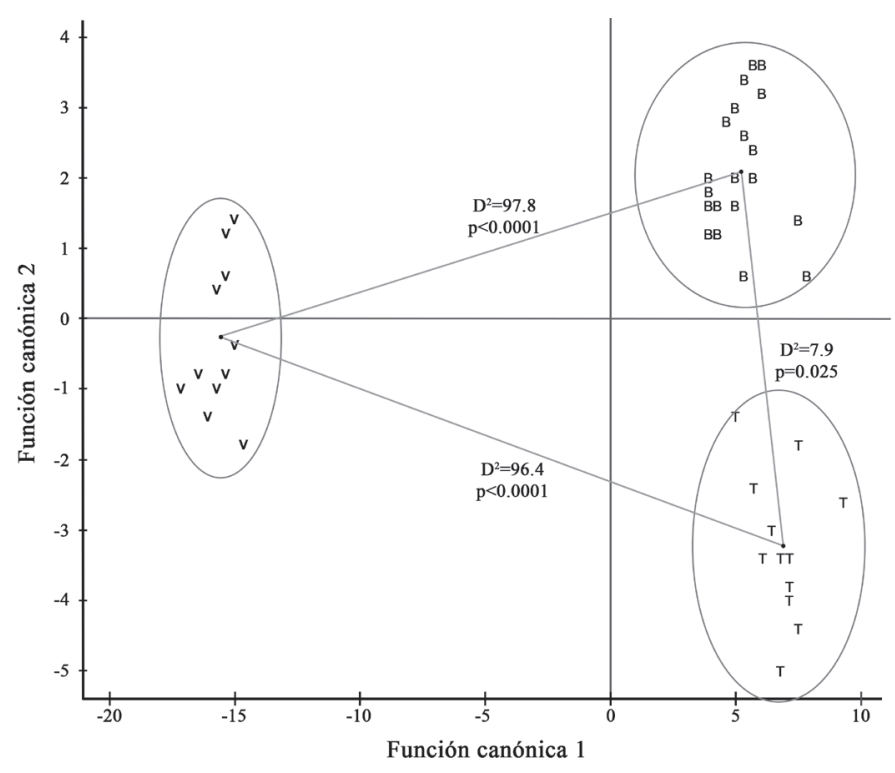

Figura 3: Agrupamiento de individuos de Agave angustifolia Haw. mediante dos funciones canónicas discriminantes y distancia de Mahalanobis (D2) entre los valores centrales de cada población estudiada en Sonora, México, V: La Vinorama, municipio Huásabas; B: El Básura, municipio Moctezuma; T: El Tepúa, municipio Aconchi.

denominado "zacatoro" en Ahuacuotzingo, Guerrero, tuvieron una densidad promedio de 5.6 \pm 3.1 individuos/100 $\mathrm{m}^{2}$; se desarrollan de 900-1403 m s.n.m., preferentemente en bosque tropical caducifolio o matorral espinoso, en pequeños manchones dispersos. Estos sitios se caracterizan por una vegetación perturbada, suelos degradados y sobreexplotación del recurso biótico (Casarrubias, 2019).

Aunque el nodricismo se reportó anteriormente (Gentry, 1982; Armenta, 2002; Barraza-Morales et al., 2006), no se había cuantificado en Sonora con poblaciones silvestres de $A$. angustifolia, las cuales parecen requerir de esta asociación. Numerosos organismos necesitan la protección de un dosel amplio y compacto de la planta nodriza, la cual protege con sombra en las horas de mayor radiación solar y a la vez permite el paso de la luz necesaria para cubrir las necesidades heliofíticas del agave (Gentry, 1972). La orientación de los agaves con respecto a la planta nodriza más cercana y/o distintiva varió, probablemente por la duración y la intensidad de la radiación solar. En Básura y Tepúa, se distribuyen principalmente hacia donde reciben la luz solar matutina; sin embargo, este patrón no se observó en Vinorama, cuando se redujo la incidencia solar vesperti- 
na debido al sistema montañoso de la Sierra La Madera. Por lo tanto, la orientación no sería crucial en esta localidad.

Un contrapreso en este hábitat xérico es la formación de redes de interacción microbioma-planta con alto potencial de especificidad y plasticidad. Las micorrizas arbusculares favorecen a través de las hifas, la transferencia de nutrientes entre plantas facilitadoras y facilitadas (Montesinos-Navarro et al., 2016), incluso aportan más nitrógeno a las especies facilitadas que poseen menos nitrógeno estructural (Montesinos-Navarro et al., 2017). Asimismo, pueden establecer reconexiones ante la ausencia de las especies con mayor afinidad, además de contrarrestar la erosión vegetal en áreas fragmentadas (Montesinos-Navarro et al., 2019).

La comunidad vegetal, particularmente las nodrizas, son esenciales para el crecimiento y desarrollo de $A$. angustifolia a través de microambientes con disponibilidad de nutrientes (Fragoso, 2011). Esto indica que la facilitación no solo debe entenderse como la modificación de las condiciones microambientales (radiación, temperatura y humedad edáfica) generada bajo el dosel. De esta manera, la actividad del microbioma compartido entre especies vegetales puede tener un papel central en la adquisición de nutrientes, proporcionando hasta $75 \%$ del fósforo y $80 \%$ del nitrógeno utilizado por las plantas (van der Heijden et al., 2008). Como consecuencia de estas interacciones se generan biofertilizantes fundamentales para su crecimiento.

La simbiosis micorrízica con el agave parece crucial en las zonas áridas donde se ha registrado una variación estacional cuantitativa y cualitativa de las especies fúngicas, que evidencian una relación funcional (Ochoa-Meza et al., 2009). En A. angustifolia de la Sierra Sonorense, la cantidad de esporas varió de $9.2 \times 10^{3}$ (invierno) a $43.5 \times 10^{3}$ (verano) por $\mathrm{dm}^{3}$, observándose 32 morfoespecies de Acaulospora Gerd. \& Trappe (10), Archaeospora J.B. Morton \& D. Redecker (2), Diversispora C. Walker \& A. Schübler (1), Glomus Tul. \& C. Tul. (17) y Pacispora Sieverd. \& Oehl. (2) (Ochoa-Meza et al., 2009). Asimismo, el análisis de correspondencia canónica realizado por Ochoa-Meza et al. (2009) mostró que las concentraciones de $\mathrm{Na}, \mathrm{K}$ y $\mathrm{Mg}$ son uno de los factores abióticos más importantes en la relación $A$. angustifolia-micorriza arbuscular.

Por otra parte, Rangel-Landa et al. (2015) observaron que las plantas nodrizas son cruciales para el establecimiento de Agave potatorum Zucc., siendo varias especies vegetales las que pueden desempeñar esta función, permitiendo una mayor germinación y supervivencia de las plántulas comparadas con los espacios abiertos. Aunque sea negativa la ganancia de carbono, la protección contra la radiación solar es un factor principal en esta asociación. De igual forma, en un estudio realizado con $A$. angustifolia, Arias-Medellín et al. (2016) documentaron que el porcentaje de germinación fue significativamente mayor en un sitio conservado vs. perturbado y favorecido por la presencia de plantas nodrizas (52.4\% germinación) vs. sitio expuesto (7.2\%). Asimismo, la supervivencia de las plántulas fue superior en las áreas no manejadas y protegidas con nodrizas.

La procedencia de las semillas es importante para el éxito de la germinación y el establecimiento temprano de las plántulas, ya que las especies de agave desarrollan diferentes mecanismos para tolerar la sequía y la baja radiación solar por acción de las nodrizas (Ramírez-Tobías et al., 2014; 2021). Por ejemplo, las plántulas de A. salmiana Otto ex Salm-Dyck dependen de estos procesos para mantener su hidratación (mayor potencial hídrico, suculencia y concentración de prolina), mientras que en $A$. striata Zucc. se conserva estable la cantidad de pigmentos (Ramírez-Tobías et al., 2014; 2021).

El nodricismo en poblaciones silvestres de $A$. angustifolia presentes en la Sierra Sonorense deberá estudiarse a mayor profundidad; establecer categorías según tamaño/ edad de los agaves, evaluar el efecto ecofisiológico en las plántulas e individuos jóvenes y maduros debido a la orientación, distancia y planta nodriza implicada (árbol, arbusto, especie).

\section{Análisis morfológico}

El promedio global de diámetro $(2.1 \pm 0.5 \mathrm{~m})$, altura $(1.4 \pm 0.3$ $\mathrm{m})$ y relación diámetro/altura $(1.5 \pm 0.3 \mathrm{~m})$ de los agaves en las tres poblaciones evaluadas están dentro de los límites registrados para la especie, incluidos sitios impactados por la actividad antropogénica en estudios previos (OchoaMeza et al., 2009; Esqueda et al., 2011). Gentry (1972) registró el diámetro de 1.5-2 m, altura de 1-1.5 m y relación diámetro/altura de $1.4 \mathrm{~m}$ sensu A. pacifica, y una década después, reportó la altura del tallo de $20-60(-90) \mathrm{cm}$, sin precisar diámetro y altura de la roseta (Gentry, 1982). Es- 
queda et al. (2011) registraron dimensiones de 0.9-2.9 $\mathrm{m}, 0.9-1.8 \mathrm{~m}$ y $1.4 \mathrm{~m}$ respectivamente. Las hojas maduras varían de lineares a lanceoladas de 50-120 × 3.5-10 cm, ascendentes a horizontales, de color verde claro a verde grisáceo (Colunga-GarcíaMarín y May-Pat, 1997; Gentry, 1972, 1982; Vázquez-García et al., 2007; Esqueda et al., 2011). En general, los especímenes tuvieron hojas más largas y anchas, biomasa foliar más elevada, dientes en mayor proporción y más largos, así como espina apical más larga y ancha, comparado con estudios previos (ColungaGarcíaMarín y May-Pat, 1997; Esqueda et al., 2011). Con ello, se amplió el rango de tamaño en algunas variables.

En un estudio realizado en Guerrero, México con $A$. angustifolia denominado "zacatoro", las variables que permitieron discriminar las poblaciones fueron: forma, borde, color y textura foliar, estrías en espinas laterales y longitud de la espina terminal. Aunque se observó una estrecha relación positiva entre el maguey zacatoro y A. angustifolia, las diferencias consistentes podrían separarlo, ayudando a brindar claridad taxonómica al género Agave (Barrientos Rivera et al., 2019).

\section{Contenido de azúcares}

La diferencia en el contenido de azúcares puede atribuirse al estado fenológico de la planta y la variación ambiental entre áreas, ya que $100 \%$ de los especímenes de Tepúa presentaba un desarrollo inicial de la inflorescencia. Diversos estudios indican que la biosíntesis y degradación dependen de las necesidades metabólicas de la planta y se inducen ante diversos factores ambientales como daño de tejidos, frío, sequía, entre otros (Valluru y Van den Ende, 2008; Versluys et al., 2018).

En especies que producen fructanos como carbohidratos de reserva, se ha encontrado que su máximo valor se observa antes de la etapa de floración, defoliación o dormancia y posteriormente se utilizan en la formación de los nuevos tejidos (Martínez-Noël y Pontis, 2000; Ritsema y Smeekens, 2003). En Agave, los fructanos se almacenan principalmente en tallos para proveer la energía necesaria en la floración y la osmoprotección ante daño y estrés (Wang y Nobel, 1998). Diferentes azúcares y fitohormonas como ácido abscísico inducen un incremento en el contenido de fructooligosacáridos en Agave tequilana y A. inaequi- dens K. Koch ante estrés abiótico (Suárez-González et al., 2014).

Para la extracción comercial de fructanos se utiliza comúnmente la raíz de chicoria (Cichorium intybus L., Asteraceae) con un contenido de $10-15 \%$. En el caso del género Agave, diversos taxones se utilizan únicamente para la producción de bebidas alcohólicas (García-Mendoza, 2012); sin embargo, representan una alternativa para la obtención de fructanos, ya que realizan una fotosíntesis tipo CAM (Crassulacean Acid Metabolism o metabolismo ácido de las crasuláceas), en la que se sintetizan principalmente estos carbohidratos (Wang y Nobel, 1998). Por ello, además de la producción de bebidas alcohólicas, $A$. tequilana y $A$. americana L. han sido consideradas con potencial para la producción de prebióticos (Franco-Robles y López, 2015; López et al., 2017). Es decir, sustancias que no son digeribles por el humano debido a su estructura molecular, como es el caso de los fructanos. Sin embargo, son empleados por la microbiota intestinal benéfica que los fermenta, estimulando así su crecimiento y con ello, favorecen la salud de los huéspedes, ya sean humanos o animales, además de contribuir en la prevención de enfermedades como cáncer y diabetes (Velázquez-Martínez et al., 2014).

El porcentaje de fructanos determinado en este trabajo establece el potencial de $A$. angustifolia para diversificar el aprovechamiento de estos azúcares. Asimismo, diferentes investigaciones se enfocan en el contenido de ART presentes en el agave como producto de la despolimerización de fructanos. Bautista-Justo et al. (2001) registraron valores entre $24-27 \%$ de ART en A. tequilana, también estimaron que entre $25-30 \%$ es adecuado para la producción de alcohol. En la Sierra Sonorense se registró un promedio de $42 \%$ de ART (Esqueda et al., 2011), mientras que en este estudio fue de $32.5 \pm 13.9 \mathrm{~g} / 100 \mathrm{~g}$ BS.

La medición de ${ }^{\circ}$ Brix es una escala arbitraria para determinar el porcentaje de sólidos solubles totales (SST) en una muestra que contiene principalmente azúcares. Los resultados del presente trabajo sugieren que la determinación de ${ }^{\circ}$ Brix podría utilizarse para estimar el contenido de fructanos y/o ART de $A$. angustifolia, un método de predicción sencillo y rápido en la cosecha de agave silvestre para la producción de bacanora. Asimismo, la base de las hojas tiene potencial de aprovechamiento como subproducto. En 
A. tequilana cultivado se han registrado correlaciones de 0.96 entre ${ }^{\circ}$ Brix y ART (Bautista-Justo et al., 2001). Existen conglomerados con mayor peso promedio del tallo y otros con menor contenido de ART que los evaluados por Esqueda et al. (2011), donde uno de ellos presentó 59.6\%. Sin embargo, la mayoría de los grupos comparten valores similares con algunas diferencias. Al parecer, la práctica empírica que realizan los productores al cortar la inflorescencia y dejar la planta una o dos temporadas más, incrementa el contenido de azúcares. El efecto de eliminar la inflorescencia y el tiempo requerido para un aumento significativo en el contenido y perfil de azúcares deberá estudiarse a profundidad.

En un análisis demográfico de dos poblaciones de A. angustifolia en un bosque tropical seco conservado y un pastizal con actividad pecuaria en Morelos, México, la simulación del corte de todas las inflorescencias provocó una $\lambda<1$, donde la población potencialmente crecería con un $\lambda>1$, disminuiría $(\lambda<1)$ o permanecería estable $(\lambda=1)$. Contrariamente, la simulación de introducción de plántulas aumentó sensiblemente en $\lambda$. Aunque la germinación y la supervivencia fueron mayores en micrositios conservados vs. deteriorados con $\lambda$ de 1.268 y 1.208 respectivamente, sugiere que esta especie puede utilizarse en programas de restauración (Arias-Medellín et al., 2016). Por lo anterior, como parte de las estrategias de conservación se propone mantener un porcentaje de los escapos florales en las poblaciones silvestres. Una experiencia exitosa de manejo de A. cupreata Trel. \& A. Berger, sugiere dejar al menos un $30 \%$ de las inflorescencias para un aporte de semillas que garantice la recuperación natural y asistida de las poblaciones (Torres et al., 2015). En A. inqequidens K. Koch se ha estimado una tasa de extracción de 10-30\% de los individuos maduros para mantener una $\lambda>1$ (Torres-García et al., 2020).

\section{Análisis canónico discriminante}

El $A C D$ mostró que dos variables fueron significativas $(p<0.01)$ y representan $100 \%$ de la varianza morfológica entre las poblaciones manejadas de Nácori Chico, con 70.8 y $29.2 \%$ para la variable 1 y 2, respectivamente (Esqueda et al., 2011). En función de los valores de los centroides obtenidos de las dos variables canónicas discriminantes, los agaves de las tres poblaciones se diferenciaron $(p<0.01)$, con distancias de Mahalanobis (D2) de 11-22. Los resultados anteriores y de la presente investigación concuerdan con los estudios previos sobre citogenética (Moreno-Salazar et al., 2007) y variabilidad genética mediante marcadores AFLP (Barraza-Morales et al., 2006; Sánchez-Teyer et al., 2009), donde las poblaciones reflejan su distancia geográfica. Debido a que los microambientes tienen poca variación entre sí, esta diferenciación observada, fortalece la hipótesis de que las poblaciones de agave se están diversificando, debido a las distancias geográficas y las barreras físicas que impiden el flujo génico, favorecidas por la competencia y el aumento en la deriva genética. Torres-García et al. (2020) consideran que los criterios ecológicos en conjunto con buenas prácticas de manejo, normativas estrictas y la organización de los productores, conducen hacia una extracción sostenible de agaves silvestres.

Aunque en las poblaciones silvestres el desarrollo de la inflorescencia es asincrónico (Gentry, 1982; MorenoSalazar et al., 2007), el color foliar y el contenido de azúcares altamente variables, este estudio permitió diferenciar las poblaciones. Asimismo, resultan indicadores de madurez sencillos de determinar en campo, facilitando la selección de individuos que cumplan con los requerimientos como materia prima. Con los resultados obtenidos en poblaciones silvestres de $A$. angustifolia en Sonora, se sugiere la recolección de especímenes que fluctúen alrededor de un tallo de $25 \mathrm{~cm}$ de diámetro con un peso de $12 \mathrm{~kg}$ y un contenido de $21 \%{ }^{\circ}$ Brix. Debido al rango amplio de $7-42 \%{ }^{\circ}$ Brix en los agaves muestreados, aún con los criterios estrictos de selección de madurez, se recomienda la prueba de sólidos solubles totales antes de la extracción. Estas consideraciones coadyuvarían en el aprovechamiento más racional del recurso en favor de la conservación de las poblaciones silvestres.

Estos criterios de selección y los resultados de estudios previos (Esqueda et al., 2013; Rangel-Landa et al., 2015; Torres et al., 2015; Arias-Medellín et al., 2016; Casarrubias, 2019; Sánchez et al., 2020; Torres-García et al., 2020) permiten la prospección de las siguientes estrategias de conservación: a) realizar un muestreo representativo de la población silvestre de agave para definir su tasa anual de extracción e introducción de plantas que permita un aprovechamiento sostenible; b) recolectar semillas, hijuelos y bulbilos para que crezcan $>25 \mathrm{~cm}$ de altura, lo cual toma de uno a dos años, bajo una superficie sombreada con riegos limitados 
para lograr plantas endurecidas; c) diseñar el trasplante de agave en agostadero, considerando los factores bióticos como el dosel de nodrizas, y abióticos del área e introducirlos al inicio de la época de lluvias; d) permitir que $30 \%$ de los individuos formen inflorescencia para que se reproduzcan sexualmente y mantengan o recuperen las poblaciones; e) cosechar el maguey hasta que alcance el tamaño y los ${ }^{\circ}$ Brix adecuados para su uso como materia prima; f) dejar crecer al maguey en áreas naturales sin riegos, fungicidas, ni insecticidas; favoreciendo así un manejo $100 \%$ orgánico para un sello verde.

\section{Conclusiones}

La densidad de agaves cambió significativamente entre las poblaciones silvestres donde se observaron agrupados en $91 \%$ y asociados a una planta nodriza en $82 \%$. El nodricismo y la eliminación de la inflorescencia deberán estudiarse con mayor detalle en un futuro con la finalidad de evaluar su papel en la conservación de Agave angustifolia. El análisis canónico discriminante permitió diferenciar las tres poblaciones silvestres de $A$. angustifolia, con similitud en sus características morfológicas y contenido de azúcares, además de establecer las características más sobresalientes durante su madurez: grado de desarrollo de la inflorescencia, color foliar y contenido de azúcares reductores totales. Los indicadores de madurez (inflorescencia, acortamiento de hojas centrales), el tamaño y el peso del tallo, y el contenido de 'Brix, coadyuvarían en la selección y aprovechamiento más racional de este agave favoreciendo su manejo sustentable.

\section{Literatura citada}

Arias-Medellín, L. A., C. Bonfil y T. Valverde. 2016. Demographic analysis of Agave angustifolia (Agavaceae) with an emphasis on ecological restoration. Botanical Sciences 94(3): 513-530. DOI: https://doi.org/10.17129/botsci.525

Armenta, A. 2002. Hongos micorrízicos y filamentosos asociados con Agave angustifolia Haw. Tesis de maestría. Centro de Investigación en Alimentación y Desarrollo A.C. Hermosillo, México. 84 pp.

Arzate-Fernández, A. M., J. L. Piña-Escutia, T. H. NormanMondragón, J. I. Reyes-Díaz, K. L. Guevara-Suárez y L. M. Vázquez-García. 2016. Regeneración de agave mezcalero (Agave angustifolia Haw.) a partir de embriones somáticos encapsulados. Revista Fitotecnia Mexicana 39(4): 359-366. DOI: https://doi.org/10.35196/rfm.2016.4.359-366

Barraza-Morales, A., F. L. Sánchez-Teyer, M. Robert, M. Esqueda y A. Gardea. 2006. Variabilidad genética en Agave angustifolia Haw. de la sierra sonorense, México, determinada con marcadores AFLP. Revista Fitotecnia Mexicana 29(1): 1-8.

Barrientos Rivera, G., E. L. Esparza Ibarra, H. R. Segura Pacheco, O. Talavera Mendoza, M. L. Sampedro Rosas y E. Hernández Castro. 2019. Caracterización morfológica de Agave angustifolia y su conservación en Guerrero, México. Revista Mexicana de Ciencias Agrícolas 10(3): 655-668. DOI: https:// doi.org/10.29312/remexca.v10i3.1554

Bautista-Justo, M., L. García-Oropeza, R. Salcedo-Hernández y L. A. Parra-Negrete. 2001. Azúcares en agaves (Agave tequilana Weber) cultivados en el estado de Guanajuato. Acta Universitaria 11(1): 33-38. DOI: https://doi.org/10.15174/ au.2001.325

Casarrubias, M. G. 2019. Uso, manejo tradicional y aspectos ecológicos del maguey zacatoro (Agave angustifolia Haw.), en el municipio de Ahuacuotzingo, Guerrero, México. Tesis de licenciatura. Universidad Autónoma de Guerrero. Chilpancingo, México. 106 pp.

Cervantes-Mendívil, T., A. D. Armenta-Calderón y J. G. Sánchez Arellano. 2007. El cultivo del maguey bacanora (Agave angustifolia Haw.) en la sierra de Sonora. Publicación Técnica No. 1. Instituto Nacional de Investigaciones Forestales Agrícolas y Pecuarias, Fundación ProduceSonora, Universidad de la Sierra. Hermosillo, México. 37 pp. Chaplin, M. F. 1986. Monosaccharides. In: Chaplin, M. F. y J. F. Kennedy (eds.). Carbohydrate analysis: a practical approach. IRL Press. Oxford, UK. Pp. 1-36.

CIE. 2018. Commission Internationale de l'Eclairage CIE 015:2018 Colorimetry. 4 ed. Vienna, Austria. DOI: https://doi. org/10.25039/TR.015.2018

Colunga-GarcíaMarín, P. y F. May-Pat. 1997. Morphological variation of henequén (Agave fourcroydes, Agavaceae) gerplasm and its wild ancestor ( $A$. angustifolia) under uniform growth conditions: diversity and domestication. American Journal of Botany 84(11): 1449-1465. DOI: https://doi.org/10.2307/2446608

Colunga-GarcíaMarín, P., E. Estrada-Loera y F. May-Pat. 1996. Patterns of morphological variation, diversity, and domestication of wild and cultivated populations of Agave in 
Yucatan, Mexico. American Journal of Botany 83(8): 126-140. DOI: https://doi.org/10.1002/j.1537-2197.1996.tb12805.x

CSRB. 2020. Consejo Sonorense Regulador del Bacanora. https://planeacion.unison.mx/noticias/nota.php?id=1968 (consultado noviembre de 2020).

Esqueda, M., A. Gutiérrez, G. Palomino, A. García-Mendoza y T. Terrazas. 2011. Morphological characterization and variation in the total content of reducing sugars in wild populations of Agave angustifolia Haw. American Journal of Agricultural and Biological Sciences 6(4): 462-468. DOI: https://doi.org/10.3844/ajabssp.2011.462.468

Esqueda, M., M. L. Coronado, A. H. Gutiérrez, T. Fragoso. 2013. Agave angustifolia Haw. Técnicas para el trasplante de vitroplantas a condiciones de agostadero. Secretaría de Agricultura Ganadería Desarrollo Rural, Pesca y Alimentación. Hermosillo, México. 18 pp.

ESRI. 2011. ArcGIS: Release 10. Environmental Systems Research Institute, Redlands. https://www.esri.com/en-us/arcgis/ products/index (consultado junio de 2021).

Fragoso, T. 2011. Factores bióticos y abióticos implicados en la distribución de poblaciones silvestres y contenido de azúcares de Agave angustifolia Haw. Tesis de maestría. Centro de Investigación en Alimentación y Desarrollo A.C. Hermosillo, México. 71 pp.

Franco-Robles, E. y M. G. López. 2015. Implication of fructans in health: Immunomodulatory and antioxidant mechanisms. The Scientific World Journal 2015: 289267. DOI: https://doi. org/10.1155/2015/289267

García-Mendoza, A. 2002. Distribution of Agave (Agavaceae) in Mexico. Cactus and Succulent Journal (US) 74(4): 177-187.

García-Mendoza, A. 2012. México, país de magueyes. La Jornada del Campo 53. https://www.jornada.com.mx/2012/02/18/ cam-pais.html (consultado octubre de 2019).

García-Mendoza, A. J., I. S. Franco y D. Sandoval. 2019. Cuatro especies nuevas de Agave (Asparagaceae, Agavoideae) del sur de México. Acta Botanica Mexicana 126: e1461. DOI: https://doi.org/10.21829/abm126.2019.1461

Gentry, S. H. 1972. The Agave Family in Sonora. Agriculture Handbook 399, United States Department of Agriculture. Washington, USA. $195 \mathrm{pp}$.

Gentry, S. H. 1982. Agaves of Continental North America. The University of Arizona Press. Tucson, USA. 670 pp.
Hintze, J. 2001. NCSS 2001. NCSS, LLC. Kaysville, USA. https:// www.ncss.com/software/ncss/ (consultado enero de 2021).

INEGI. 2001. Carta Edafológica H12-8, H12-9, G12-03. 1:250,000 (Vectorial). Serie I. Instituto Nacional de Estadística, Geografía e Informática, México. https://www.inegi.org.mx/temas/ edafologia/\#Descargas (consultado junio de 2021)

INEGI. 2008. Colección Cartas Climatológicas Escalas 1:250,000 y 1:1000,000 (Vectorial). Instituto Nacional de Estadística, Geografía e Informática, México. https://www.inegi.org.mx/ temas/climatologia/\#Descargas (consultado junio de 2021).

López, M. G., R. M. Camacho-Ruíz, M. González-Ávila, E. MelladoMojica, L. Moreno-Vilet, C. I. Godínez-Hernández, J. R. Aguirre-Rivera, M. de la Mora-Amutio, B. I. Juárez-Flores, G. Ramos-Clamont, R. I. Armenta-Corral, R. Prado-Ramírez, M. A. Mendoza-Rivera, J. Arrizon, I. Andrade-González, P. I. Aldrete-Herrera y R. I. Ortiz-Basurto. 2017. Fructanos de agave: actualidad y perspectivas. In: Gschaedler, A. C. (ed.). Panorama del aprovechamiento de los agaves en México. Consejo Nacional de Ciencia y Tecnología (México), Centro de Investigación y Asistencia en Tecnología y Diseño del Estado de Jalisco A.C., Red Temática Mexicana Aprovechamiento Integral Sustentable y Biotecnología de los Agaves. Guadalajara, México. Pp. 69-120.

Martínez-Noël, G. y H. G. Pontis. 2000. Involvement of sucrose synthase in sucrose synthesis during mobilization of fructans in dormant Jerusalem artichoke tubers. Plant Science 159(2): 191-195. DOI: https://doi.org/10.1016/ s0168-9452(00)00317-4

Megazyme. 2011. Megazyme Dietary Fiber Measurement. Pp. 19. https://prod-media.megazyme.com/media/pdf/86/b3/f2/ df-brochure-full-brochure.pdf (consultado junio de 2021).

Montesinos-Navarro, A., M. Verdú, J. I. Querejeta y A. ValienteBanuet. 2017. Nurse plants transfer more nitrogen to distantly related species. Ecology 98(5): 1300-1310. DOI: https://doi.org/10.1002/ecy.1771

Montesinos-Navarro, A., G. Díaz, P. Torres, F. Caravaca y A. Roldán. 2019. Phylogenetic rewiring in mycorrizal-plant interaction networks increases community stability in naturally fragmented landscapes. Communications Biology 2: 452. DOI: https://doi.org/10.1038/s42003-019-0700-3

Montesinos-Navarro, A., M. Verdú, J. I. Querejeta, L. Sortibrán y A. Valiente-Banuet. 2016. Soil fungi promote nitrogen 
transfer among plants involved in long-lasting facilitative interactions. Perspectives in Plant, Ecology, Evolution and Systematics 18: 45-51. DOI: https://doi.org/10.1016/j. ppees.2016.01.004

Moreno-Salazar, S. F., G. Palomino, J. Martínez y M. Esqueda. 2007. Tamaño del genoma y cariotipo en Agave angustifolia y $A$. rhodacantha de Sonora, México. Revista Fitotecnia Mexicana 30: 23-33.

Ochoa-Meza, A., M. Esqueda, R. Fernández-Valle y R. Herrera-Peraza. 2009. Variación estacional de hongos micorrízicos arbusculares asociados con Agave angustifolia Haw. en la sierra sonorense, México. Revista Fitotecnia Mexicana 32: 189-199.

Ramírez-Tobías, H. M., R. A. Cedillo de la Rosa, J. Flores y C. LópezPalacios. 2021. Seedlings from two Agave species differing in microhabitat evolve different tolerance mechanisms to drought and shade under nurse plants. Flora 274: 151729. DOI: https://doi.org/10.1016/j.flora.2020.151729

Ramírez-Tobías, H. M., C. B. Peña-Valdivia y J. R. Aguirre. 2014. Respuestas bioquímico-fisiológicas de especies de Agave a la restricción de humedad. Botanical Sciences 92(1): 131-139. DOI: https://doi.org/10.17129/botsci.156

Rangel-Landa, S., A. Casas y P. Dávila. 2015. Facilitation of Agave potatorum: An ecological approach for assisted population recovery. Forest Ecology and Management 347: 57-74. DOI: https://doi.org/10.1016/j.foreco.2015.03.003

Ritsema, T. y S. Smeekens. 2003. Fructans: Beneficial for plants and humans. Current Opinion in Plant Biology 6(3): 223-230. DOI: https://doi.org/10.1016/s1369-5266(03)00034-7

Rivera-Lugo, M., A. García-Mendoza, J. Simpson, E. Solano, K. GilVega. 2018. Taxonomic implications of the morphological and genetic variation of cultivated and domesticated populations of the Agave angustifolia complex (Agavoideae, Asparagaceae) in Oaxaca, Mexico. Plant Systematics and Evolution 304(8): 969-979. DOI: https://doi.org/10.1007/ s00606-018-1525-0

Rodríguez-Garay, B., J. A. Lomelí-Sención, E. Tapia-Campos, A. Gutiérrez-Mora, J. García-Galindo, J. M. RodríguezDomínguez, D. Urbina-López e I. Vicente-Ramírez. 2009. Morphological and molecular diversity of Agave tequilana Weber var. Azul and Agave angustifolia Haw. var. Lineño. Industrial Crops and Products 29(1): 220-228. DOI: https:// doi.org/10.1016/j.indcrop.2008.05.007
Salazar, V., J. Moreno y E. Casas. 2015. Innovación para el fomento de la competitividad en el proceso artesanal de producción de bacanora. Estudios Sociales 23(46): 214-240.

Sánchez, A., Z. Coronel-Lara, A. Gutiérrez, G. Vargas, M. L. Coronado y M. Esqueda. 2020. Aclimatación y trasplante de vitroplantas de Agave angustifolia Haw. en condiciones silvestres. Revista Mexicana de Ciencias Agrícolas 11(7): 1593-1605. DOI: https://doi.org/10.29312/remexca.v11i7.2403

Sánchez-Teyer, F., S. Moreno-Salazar, M. Esqueda, A. Barraza y M. L. Robert. 2009. Genetic variability of wild Agave angustifolia populations based on AFLP: a basic study for conservation. Journal of Arid Environments 73(6-7): 611616. DOI: https://doi.org/10.1016/j.jaridenv.2009.01.008

SAS. 2008. SAS/STAT v. 9.2 user's guide. SAS Institute Inc. Cary, USA. https://www.sas.com/es_mx/software/stat.html (consultado enero de 2021).

Suárez-González, E. M., M. G. López, J. P. Délano-Frier y J. F. GómezLeyva. 2014. Expression of the 1-SST and 1-FFT genes and consequent fructan accumulation in Agave tequilana and A. inaequidens is differentially induced by diverse (a)bioticstress related elicitors. Journal of Plant Physiology 171(3-4): 359-372. DOI: https://doi.org/10.1016/j.jplph.2013.08.002 Torres, I., A. Casas, E. Vega, M. Martínez-Ramos y A. DelgadoLemus. 2015. Population dynamics and sustainable management of mescal agaves in Central Mexico: Agave potatorum in the Tehuacán-Cuicatlán Valley. Economic Botany 69(1): 26-41. DOI: https://doi.org/10.1007/s12231014-9295-2

Torres-García, I., A. León-Jacinto, E. Vega, A. I. Moreno-Calles y A. Casas. 2020. Integral projection models and sustainable forest management of Agave inaequidens in Western Mexico. Frontiers in Plant Science 11: 1224. DOI: https:// doi.org/10.3389/fpls.2020.01224

Valluru, R. y W. Van den Ende. 2008. Plant fructans in stress environments: emerging concepts and future prospects. Journal of Experimental Botany 59(11): 2905-2916. DOI: https://doi.org/10.1093/jxb/ern164

van der Heijden, M. G. A., R. D. Bardgett y N. M. van Straalen. 2008. The unseen majority: soil microbes as drivers of plant diversity and productivity in terrestrial ecosystems. Ecology Letters 11(3): 296-310. DOI: https://doi.org/10.1111/ j.1461-0248.2007.01139.x 
Vázquez-García, J. A., M. J. Cházaro-Basáñez, G. HernándezVera, Y. L. Vargas-Rodríguez y M. P. Zamora-Tavares. 2007. Taxonomía del género Agave (Agavaceae) en el occidente de México: una panorámica preliminar. In: Vázquez-García, J. A., M. J. Cházaro-Basáñez, G. Hernández-Vera y E. FloresBerrios (eds.). Agaves del occidente de México. Universidad de Guadalajara. Guadalajara, México. Pp. 145-191.

Velázquez-Martínez, J. R., R. M. González-Cervantes, M. A Hernández-Gallegos, R. Campos, A. R. Jiménez y M. L. Arenas. 2014. Prebiotic potential of Agave angustifolia Haw. fructans with different degrees of polymerization.
Molecules 19(8): 12660-12675. DOI: https://doi. org/10.3390/molecules190812660

Versluys, M., O. Kirtel, E. Toksoy Öner y W. Van den Ende. 2018. The fructan syndrome: Evolutionary aspects and common themes among plants and microbes. Plant Cell and Environment 41(1): 16-38. DOI: https://doi.org/10.1111/ pce. 13070

Wang, N. y P. S. Nobel. 1998. Phloem transport of fructans in the crassulacean acid metabolism species Agave deserti. Plant Physiology 116(2): 709-714. DOI: https://doi.org/10.1104/ pp.116.2.709 